\title{
The genetic backgrounds in nonalcoholic fatty liver disease
}

\author{
Yuya Seko $\cdot$ Kanji Yamaguchi $^{1} \cdot$ Yoshito Itoh $^{1}$
}

Received: 14 February 2018 / Accepted: 22 February 2018 / Published online: 28 February 2018

(c) Japanese Society of Gastroenterology 2018

\begin{abstract}
Nonalcoholic fatty liver disease (NAFLD) is the most prevalent chronic liver disease worldwide. Nonalcoholic steatohepatitis (NASH), a severe form of NAFLD, can lead to hepatocellular carcinoma (HCC) and hepatic failure. The development and progression of NAFLD are determined by environmental and genetic factors. The effect of genetic factors has been demonstrated by familial studies, twin studies and several cross-sectional studies. In the past 10 years, genome-wide association studies have revealed several single nucleotide polymorphisms (SNPs) associated with the pathology of NAFLD. Among them, the Patatin-like phospholipase domain-containing 3 (PNPLA3) gene variant I148M showed a strong relationship with the development and progression of NAFLD, NASH, and NAFLD-related HCC. The transmembrane 6 superfamily member 2 (TM6SF2) gene variant E167 K was also associated with NAFLD, and it has a relationship with cardiovascular disease. Furthermore, several genes have been proposed as candidate genes to be associated with NAFLD based on case-control studies. We conducted a comprehensive literature search and review on the genetic background of NAFLD.
\end{abstract}

Keywords Nonalcoholic fatty liver disease (NAFLD) - Genome-wide association study (GWAS) - PNPLA3 · TM6SF2 . Glucokinase regulator $(G C K R)$

\section{Introduction}

Nonalcoholic fatty liver disease (NAFLD) is the most common cause of chronic liver disease and results in serious public health problems. Globally, about $25 \%$ of the adult population is estimated to suffer from NAFLD [1]. NAFLD has a wide spectrum of liver pathology, ranging from nonalcoholic fatty liver (NAFL), which is usually benign, to nonalcoholic steatohepatitis (NASH), which is characterized by steatosis plus features of cellular injury, such as inflammation and hepatocyte ballooning, and may progress to liver cirrhosis (LC), hepatic failure and hepatocellular carcinoma (HCC) in the absence of significant alcohol consumption [2, 3]. NASH is now the second indication [4] and is predicted to become the main indication for liver transplantation in the United States by 2020 [5]. The most common cause of death in patients with NAFLD is cardiovascular disease, followed

Yuya Seko

yuyaseko@koto.kpu-m.ac.jp

1 Molecular Gastroenterology and Hepatology, Kyoto Prefectural University of Medicine, 465 Kajii-cho, Kawaramachi-Hirokoji, Kamigyo-ku, Kyoto 602-8566, Japan by non-liver malignancy and liver-related death [6-8]. It has become clear that patients with NASH, especially those with advanced fibrosis, are a high-risk group for HCC and death from liver-related causes. The risks for disease progression and development of NAFLD depend on environmental factors and multiple genetic factors.

NAFLD is strongly associated with metabolic syndrome, including type 2 diabetes mellitus (T2DM), dyslipidemia, and obesity. Because of a lifestyle of high-calorie food intake and lack of exercise, the prevalence of obesity and insulin resistance are increasing. Some dietary habits may influence the progression of NAFLD, such as monounsaturated fatty acid consumption [9], fructose consumption [10, 11], and coffee consumption [12-14]. Furthermore, some studies reported that the intestinal microbiome may influence NAFLD development $[15,16]$.

On the other hand, some familial and twin studies have supported a heritable effect of NAFLD [17-19]. Among monozygotic twins, the concordance of disease severity, including the degree of fibrosis and steatosis, was greater than that among dizygotic twins. The prevalence of NAFLD also differs among ethnic groups. NAFLD is most common in East Asian Indians, followed by Hispanics, Asians, Caucasians, and less frequently, African Americans [20-22]. 
These genetic backgrounds affect the pathology of NAFLD directly or via variability in metabolism and wound healing response. Understanding the contribution of genetic background may have relevance not only to surveillance and therapeutic strategies but also to preventative measures for developing NAFLD. In this review, we have tried to summarize the literature on the role of genetics in NAFLD.

\section{GWAS on NAFLD}

Genome-wide association studies (GWAS) are used to identify the association between common variants in arrays and polymorphic diseases. Because the GWAS approach is called a "hypothesis-free method", it can identify unreported genes or biologic pathways. On the other hand, we cannot always understand how identified loci contribute to disease progression. Furthermore, GWAS cannot check all of the genetic factors associated with a disease, and the results depend on the subjects in the study. However, GWAS are more likely to identify genetic risk than candidate gene studies.

Romeo et al. first reported in 2008 that rs 738409 C > G SNP in the Patatin-like phospholipase domain-containing
3 (PNPLA3) gene was significantly associated with liver steatosis [23]. After that study, several GWAS have been performed in different populations and have identified phenotypes [24-31] (Table 1). The identified SNPs were different depending on the population studied and the phenotype. The PNPLA3 rs738409 SNP showed a strong relationship with the development of NAFLD or NASH, hepatic steatosis and hepatic fibrosis in almost all studies. There were 2 studies analyzed the association of genetic risk with liver histology in Japanese population [26, 27]. NAFLD was diagnosed by liver biopsy in both studies. Kawaguchi et al. recruited 529 histologically diagnosed NAFLD patients and 932 population controls. They found that PNPLA3 rs738409 showed strong association with Matteoni type 4 subgroup $(P=1.7610216, \mathrm{OR}=2.18,95 \%$ CI 1.81-2.63). Kitamoto et al. analyzed 392 biopsy-proven NAFLD subjects and 934 control individuals. PNPLA3 rs738409 was most strongly associated with NAFLD after adjusted by age, gender, and BMI $(P=6.8910-14$, $\mathrm{OR}=2.05)$. Based on these GWAS, PNPLA3 rs738409 and Transmembrane 6 superfamily member 2 (TM6SF2) rs58542926 are thought to be the main contributors to NAFLD pathogenesis and progression.

Table 1 Genome-wide association studies of non-alcohol fatty liver disease

\begin{tabular}{|c|c|c|c|c|c|}
\hline Study & Population & Subjects (N) & SNPs & Phenotype & Gene(s) \\
\hline Romeo S et al. [23] & Multi ethnic & 2051 & 9229 & Hepatic fat content & PNPLA3 rs738409 \\
\hline Chalasani et al. [24] & Non-Hispanic white women & 236 & 324,623 & $\begin{array}{l}\text { NAS, } \\
\text { hepatic fibrosis, } \\
\text { lobular inflammation, } \\
\text { ALT }\end{array}$ & $\begin{array}{l}\text { FDFT1 rs2645424 } \\
\text { PDGFA rs343062 } \\
\text { COL13A1 rs1227756, LTBP3 } \\
\text { rs6591182, EFCAB4B } \\
\text { rs887304 } \\
\text { rs2499604, PZP rs6487679, } \\
\text { rs } 1421201,2710833\end{array}$ \\
\hline Speliotes et al. [25] & Multi ethnic & $\begin{array}{l}7176 \\
592 \text { (biopsy) }\end{array}$ & -2.4 million & $\begin{array}{l}\text { Steatosis by CT } \\
\text { histological NAFLD }\end{array}$ & $\begin{array}{l}\text { PNPLA3 rs738409, NCAN } \\
\text { rs2228603, PPP1R3B } \\
\text { rs4240624 } \\
\text { PNPLA3 rs738409, NCAN } \\
\text { rs2228603, GCKR rs780094, } \\
\text { LYPLAL1 rs12137855 }\end{array}$ \\
\hline Kawaguchi et al. [26] & Japanese & 529 & 484,751 & Matteoni type 4 & PNPLA3 rs 738409 \\
\hline Kitamoto et al. [27] & Japanese & 392 & 261,540 & $\begin{array}{l}\text { NAFLD, steatosis, fibrosis, } \\
\text { NAS }\end{array}$ & $\begin{array}{l}\text { PNPLA3 rs738409, SAMM50 } \\
\text { rs3761472, PARVB } \\
\text { rs6006611 }\end{array}$ \\
\hline Feitosa et al. [28] & Caucasian & 2705 & -2.5 million & Steatosis by $\mathrm{CT}$ & $\begin{array}{l}\text { PNPLA3 rs738409, PPP1R3B } \\
\text { rs2126259, ERLIN-CHUK- } \\
\text { CWF19L1 gene cluster }\end{array}$ \\
\hline Kozlitina et al. [29] & Multi ethnic & 4625 & 247,870 & $\begin{array}{l}\text { Hepatic fat content by }{ }^{1} \mathrm{H}- \\
\text { MRS }\end{array}$ & $\begin{array}{l}\text { PNPLA3 rs738409, TM6SF2 } \\
\text { rs58542926 }\end{array}$ \\
\hline DiStefano et al. [30] & Multi ethnic & 2300 & 605,718 & Hepatic fat content & $\begin{array}{l}\text { PNPLA3 rs } 738409, \text { SUGP1 } \\
\text { rs10401969 }\end{array}$ \\
\hline Adams et al. [31] & Australian adolescent & 928 & $2,078,505$ & NAFLD by US & $\begin{array}{l}\text { LPPR4 rs12743824, GC } \\
\text { rs222054, LCP1 rs7324845, } \\
\text { SLC38A8 rs11864146 }\end{array}$ \\
\hline
\end{tabular}




\section{PNPLA3}

PNPLA3, also called adiponutrin is the most robust genetic variant associated with NAFLD. The first GWAS revealed the association of a PNPLA3 variant with hepatic fat content [23]. The result was validated by other GWAS independently, and many candidate studies in various ethnic groups clarified the association with histological severity, including hepatic fibrosis [32-34]. This relationship has been reported in pediatric patients as well, such as Hispanic, Taiwanese, and Caucasian children and adolescents [35-37]. The PNPLA3 variant also confers an increased risk of NAFLD-related HCC in European and Japanese populations [38, 39]. This hepatocarcinogenetic effect has been reported not only in patients with NAFLD, but also in patients with alcohol-related disease and chronic viral hepatitis in a meta-analysis [40]. These effects of the I148M substitution were independent from obesity, T2DM, and insulin resistance [41, 42]. In an animal model and in human hepatocytes, PNPLA3 activity is regulated by glucose and insulin via pathways involving the sterol regulatory element binding protein 1-c [43]. The I148M substitution leads to loss of enzymatic activity of hydrolyzed triglycerides and retinyl esters, resulting in the accumulation of triglycerides and esters in lipid droplets of hepatocytes and hepatic stellate cells up to twofold greater than with wild type [44-46]. It also induces hepatic inflammation and fibrosis. Moreover, carriers of the I148M variant have lower levels of adiponectin, which has an anti-inflammatory effect [47] and inhibits the activation of pro-fibrotic hepatic stellate cells [48, 49]. In contrast, no study has reported an association of PNPLA3 with other fibrogenic factors, such as TNF, $\alpha$-smooth muscle actin, and type 1 collagen. In an animal model, the PNPLA3 deletion produced no change in phenotype; however, overexpression and knock-in mutations led to increased hepatic fat concentrations [50]. These findings suggest the hypothesis that the accumulation of the I148M mutation on droplets escapes degradation by the proteasome and inhibits the activity of PNPLA3 in hepatocytes [50]. Other studies also revealed that the I148M variant was associated with the response to lifestyle change [51, 52]. In that study, researchers found that liver fat content decreased significantly more in the PNPLA3 genotype GG group than in the PNPLA3 genotype CC group after a short course of a low carbohydrate diet, although patients of both genotypes lost similar amounts of body weight [51]. A recent study proposed that the downregulation of the I148M mutation could be a therapeutic target for NAFLD [53]. Additional studies are warranted to determine the utility of PNPLA3 rs738409 in risk classification of NASH and NAFLDrelated $\mathrm{HCC}$.

\section{TM6SF2}

The TM6SF2 variant was shown to associate with hepatic fat content by Kozlitina et al. [29]. A validation study revealed the association of TM6SF2 with hepatic fibrosis [54]. In that study, carriers of the TM6SF2 E167K mutation had 1.9-fold increased risk of advanced fibrosis independent of the PNPLA3 genotype. However, a relationship between TM6SF2 E167K and NAFLD was not reported in the Japanese population. Though a plausible reason for this was not clear, the discrepancy may be based on the difference in minor allele frequency and inter-ethnic variation or an underpowered cohort. TM6SF 2 is involved in the secretion of very low-density lipoprotein from the hepatocyte. It concerns the triglyceride to apolipoprotein B100 pathway. The E167K mutation leads to loss of this function and results in increasing liver triglyceride content and decreasing circulating lipoproteins. It is well known that carriers of the E167K mutation tend to have greater risk of fatty liver, but a lower risk of cardiovascular disease [29, 54-56]. Kozlitina et al. [29] identified the association of the TM6SF2 variant with increased hepatic triglyceride content, a reduced serum triglyceride level, and low-density lipoprotein cholesterol. They hypothesized that TM6SF2 regulated not only hepatic lipoprotein secretion, but also hepatic synthesis of triglycerides. Carriers of the E167K form had an approximately $50 \%$ lower risk of developing atherosclerotic carotid plaques, and a $40 \%$ reduction in the risk of cardiovascular events in Western countries [56]. At present, no study has determined whether the TM6SF2 variant affects the risk of NAFLD-related HCC. Further study regarding the association between TM6SF2 and the risk of HCC in the pathogenesis of NAFLD is needed.

\section{GCKR}

Glucokinase regulator $(G C K R)$ regulates the flow of glucose in hepatocytes and de novo lipogenesis. The missense mutation (P446L) of GCKR rs 1260326 was associated with liver fat content in NAFLD [57]. The P446L mutation leads to a reduction in the ability of glucokinase in response to fructose-6-phosphate, and results in hepatic glucose intake [58]. This mutation also leads to a decrease in the serum level of glucose and insulin, but an increase in malonyl Co-A, which is used as substrate for hepatic lipogenesis and blocks fatty acid $\beta$-oxidation. This pathway increases hepatic fat accumulation. Recently, several studies reported that mutation of GCKR affects not only lipogenesis and liver fat content, but also hepatic fibrosis in patients with NAFLD [59, 60]. This result was confirmed by a recent meta-analysis [61] that revealed the association of GCKR with NAFLD-related fibrosis in both the Asian and non-Asian populations. 


\section{Other genetic variants associated with metabolism}

Several genes were identified in cross-sectional or case-control studies as potential candidate genes associated with NAFLD. Table 2 shows the function and phenotype of these candidate genes. There are several candidate genes reported besides those in Table 2. In this review, we chose to discuss four genes associated with insulin signaling and lipid metabolism.

\section{Ectonucleotide pyrophosphatase/ phosphodiesterase 1 (ENPP1)}

\section{Insulin receptor substrate 1 (IRS1)}

Insulin resistance plays a key role in the pathology of NAFLD. That is a common feature of NAFLD, T2DM, and metabolic syndrome. The genes involved in hepatic insulin signaling have been reported to be associated with NAFLD. The ENPP1 rs1044498 K121Q mutation leads to an interaction between ENPP1 and the insulin receptor, inhibiting signaling. It was also reported that the IRSI rs 1801278 G972R variant was associated with decreased activity of IRS-1, thereby reducing insulin signaling in the liver. A large study that included 702 biopsy-proven NAFLD patients found that both ENPPI and IRS- 1 SNPs were associated with a reduction in insulin signaling activity and severe fibrosis [62]. These ENPPI and IRSI variants promoted insulin resistance by reducing AKT activation [62].

\section{Peroxisome proliferative activated receptor (PPAR)}

\section{Lpin1}

Peroxisome proliferator-activated nuclear receptors (PPAR) play a role in hepatic lipid metabolism. PPAR- $\alpha$ acts as a molecular sensor for long-chain fatty acids, eicosanoids, and fibrates by upregulation of mitochondrial uptake and $\beta$-oxidation. PPAR- $\alpha$ activation improves steatosis, inflammation, and fibrosis in NAFLD [63]. Chen et al. [64] reported the association of the PPAR- $\alpha$ V227A variant with NAFLD. They described that the V227 isoform has lower activity than the A227isoform, resulting in reduced lipid catabolism and an increased risk of NAFLD. PPAR $\gamma$ improves insulin resistance and has been reported to restore adipose tissue insulin sensitivity and decrease free fatty acid flux to the liver [65]. PPAR- $\gamma$ rs1805192 P12A leads to loss of function of the gene product, impairing its ability to bind DNA and activate transcription. This variant decreases sensitivity to insulin and anti-inflammatory effects. However, the association of PPARs with the pathology of NAFLD remains controversial $[66,67]$.

LPIN1 is expressed mainly in liver and adipose tissue. LPIN1 plays a role in the synthesis of phospholipids and triglycerides, and regulates fatty acid metabolism [68]. A previous study reported that carriers of the LPIN1 rs 13412852 TT genotype showed increased expression of LPIN1 and had a smaller risk of NAFLD [69]. Variants of LIPINI influence the phenotype not only of NAFLD, but also of other metabolic traits [70].

\section{Conclusion}

In this review, we found that several genes affect the development, histological changes, progression, and carcinogenesis of NAFLD in various manners. At present, the PNPLA3 gene variant is the most powerful and validated genetic factor for steatosis, fibrosis, disease progression, and HCC in multiple ethnic groups. These result support the hypothesis that PNPLA3 acts as a main player of disease progression in combination with a high-calorie diet or alcohol consumption. However, we cannot understand the pathology of NAFLD by only one gene. We must classify the risk of NAFLD by combining the potential effects of the risk genes in each patient. Further studies about how these risk genes influence the pathology of NAFLD are needed. Such
Table 2 Function and phenotype of candidate genes associated with non-alcohol fatty liver disease

\begin{tabular}{lll}
\hline Gene & Function & Phenotype \\
\hline PNPLA3 rs738409 & Lipid droplet content in hepatocyte & Steatosis, fibrosis, NASH, HCC \\
TM6SF2 rs58542926 & VLDL secretion & Fibrosis, NAFLD, NASH \\
LYPLAL1 rs12137855 & TG catabolism & NAFLD \\
GCKR rs780094 & de novo lipogenesis & Fibrosis, NAFLD, NASH \\
LPIN1 rs13412852 & Lipid metabolism & Fibrosis, NASH \\
ENPP1 rs1044498 & Insulin signaling inhibitor & Fibrosis \\
IRS1 rs1801278 & Insulin signaling & Fibrosis \\
PPAR $\alpha$ rs1800206 & Lipid metabolism & Steatosis, inflammation, fibrosis \\
\hline
\end{tabular}


information will help us define surveillance approaches and therapeutic strategies, including lifestyle interventions and pharmacological therapies.

\section{Compliance with ethical standards}

Conflict of interest Yuya Seko, Kanji Yamaguchi, and Yoshito Itoh declare that they have no conflict of interest.

Human rights This study does not involve any data about human subjects.

Informed consent This study does not involve human subjects.

\section{References}

1. Younossi ZM, Koenig AB, Abdelatif D, et al. Global epidemiology of nonalcoholic fatty liver disease-Meta-analytic assessment of prevalence, incidence, and outcomes. Hepatology. 2016;64:73-84.

2. Chalasani N, Younossi Z, Lavine JE, et al. The diagnosis and management of non-alcoholic fatty liver disease: practice guideline by the American Association for the Study of Liver Diseases, American College of Gastroenterology, and the American Gastroenterological Association. Hepatology. 2012;55:2005-23.

3. Watanabe S, Hashimoto E, Ikejima K, et al. Japanese Society of Gastroenterology; Japan Society of Hepatology. Evidence based clinical practice guidelines for nonalcoholic fatty liver disease/ nonalcoholic steatohepatitis. J Gastroenterol. 2015;50:364-77.

4. Wong RJ, Aguilar M, Cheung R, et al. Nonalcoholic steatohepatitis is the second leading etiology of liver disease among adults awaiting liver transplantation in the United States. Gastroenterology. 2015;148:547-55.

5. Charlton MR, Burns JM, Pedersen RA, et al. Frequency and outcomes of liver transplantation for nonalcoholic steatohepatitis in the United States. Gastroenterology. 2011;141:1249-53.

6. Ong JP, Pitts A, Younossi ZM. Increased overall mortality and liver-related mortality in non-alcoholic fatty liver disease. J Hepatol. 2008;49:608-12.

7. Rafiq N, Bai C, Fang Y, et al. Long-term follow-up of patients with nonalcoholic fatty liver. Clin Gastroenterol Hepatol. 2009;7:234-8.

8. Angulo P, Bugianesi E, Bjornsson ES, et al. Simple noninvasive systems predict long-term outcomes of patients with nonalcoholic fatty liver disease. Gastroenterology. 2013;145:782-9.

9. Parker HM, Johnson NA, Burdon CA, et al. Omega-3 supplementation and non-alcoholic fatty liver disease: a systematic review and meta-analysis. J Hepatol. 2012;56:944-51.

10. Abid A, Taha O, Nseir W, et al. Soft drink consumption is associated with fatty liver disease independent of metabolic syndrome. J Hepatol. 2009;51:918-24.

11. Abdelmalek MF, Suzuki A, Guy C, et al. Increased fructose consumption is associated with fibrosis severity in patients with nonalcoholic fatty liver disease. Hepatology. 2010;51:1961-71.

12. Saab S, Mallam D, Cox GA 2nd, et al. Impact of coffee on liver diseases: a systematic review. Liver Int. 2014;34:495-504.

13. Molloy JW, Calcagno CJ, Williams CD, et al. Association of coffee and caffeine consumption with fatty liver disease, nonalcoholic steatohepatitis, and degree of hepatic fibrosis. Hepatology. 2012;55:429-36.

14. Klatsky AL, Morton C, Udaltsova N, et al. Coffee, cirrhosis, and transaminase enzymes. Arch Intern Med. 2006;166:1190-5.
15. Zhao L. Genomics: the tale of our other genome. Nature. 2010;465:879-80.

16. Quigley EM, Monsour HP. The gut microbiota and nonalcoholic fatty liver disease. Semin Liver Dis. 2015;35:262-9.

17. Willner IR, Waters B, Patil SR, et al. Ninety patients with nonalcoholic steatohepatitis: insulin resistance, familial tendency, and severity of disease. Am J Gastroenterol. 2001;96:2957-61.

18. Struben VM, Hespenheide EE, Caldwell SH. Nonalcoholic steatohepatitis and cryptogenic cirrhosis within kindreds. Am J Med. 2000;108:9-13.

19. Schwimmer JB, Celedon MA, Lavine JE, et al. Heritability of nonalcoholic fatty liver disease. Gastroenterology. 2009;136:1585-92.

20. Hernaez R. Genetic factors associated with the presence and progression of nonalcoholic fatty liver disease: a narrative review. Gastroenterol Hepatol. 2012;35:32-41.

21. Zimmer V, Lammert F. Genetics and epigenetics in the fibrogenic evolution of chronic liver diseases. Best Pract Res Clin Gastroenterol. 2011;25:269-80.

22. Duvnjak M, Barsić N, Tomasić V, Lerotić I. Genetic polymorphisms in non-alcoholic fatty liver disease: clues to pathogenesis and disease progression. World J Gastroenterol. 2009;15:6023-7.

23. Romeo S, Kozlitina J, Xing C, et al. Genetic variation in PNPLA3 confers susceptibility to nonalcoholic fatty liver disease. Nat Genet. 2008;40(12):1461-5.

24. Chalasani N, Guo X, Loomba R, et al. Genome-wide association study identifies variants associated with histologic features of nonalcoholic Fatty liver disease. Gastroenterology. 2010;139(5):1567-76 (1576.e1-6).

25. Speliotes EK, Yerges-Armstrong LM, Wu J, et al. Genome-wide association analysis identifies variants associated with nonalcoholic fatty liver disease that have distinct effects on metabolic traits. PLoS Genet. 2011;7(3):e1001324.

26. Kawaguchi T, Sumida Y, Umemura A, et al. Genetic polymorphisms of the human PNPLA3 gene are strongly associated with severity of non-alcoholic fatty liver disease in Japanese. PLoS One. 2012;7(6):e38322.

27. Kitamoto T, Kitamoto A, Yoneda M, et al. Genome-wide scan revealed that polymorphisms in the PNPLA3, SAMM50, and PARVB genes are associated with development and progression of nonalcoholic fatty liver disease in Japan. Hum Genet. 2013;132(7):783-92.

28. Feitosa MF, Wojczynski MK, North KE, et al. The ERLIN1CHUK-CWF19L1 gene cluster influences liver fat deposition and hepatic inflammation in the NHLBI Family Heart Study. Atherosclerosis. 2013;228(1):175-80.

29. Kozlitina J, Smagris E, Stender S, et al. Exome-wide association study identifies a TM6SF2 variant that confers susceptibility to nonalcoholic fatty liver disease. Nat Genet. 2014;46(4):352-6.

30. DiStefano JK, Kingsley C, Craig Wood G, et al. Genome-wide analysis of hepatic lipid content in extreme obesity. Acta Diabetol. 2015;52(2):373-82.

31. Adams LA, White SW, Marsh JA, et al. Association between liverspecific gene polymorphisms and their expression levels with nonalcoholic fatty liver disease. Hepatology. 2013;57(2):590-600.

32. Valenti L, Al-Serri A, Daly AK, et al. Homozygosity for the patatin-like phospholipase-3/adiponutrin I148M polymorphism influences liver fibrosis in patients with nonalcoholic fatty liver disease. Hepatology. 2010;51:1209-17.

33. Sookoian S, Castano GO, Burgueno AL, et al. A nonsynonymous gene variant in the adiponutrin gene is associated with nonalcoholic fatty liver disease severity. J Lipid Res. 2009;50:2111-6.

34. Rotman Y, Koh C, Zmuda JM, et al. The association of genetic variability in patatin-like phospholipase domain containing protein 3 (PNPLA3) with histological severity of nonalcoholic fatty liver disease. Hepatology. 2010;52:894-903. 
35. Goran MI, Walker R, Le KA, et al. Effects of PNPLA3 on liver fat and metabolic profile in Hispanic children and adolescents. Diabetes. 2010;59:3127-30.

36. Lin YC, Chang PF, Hu FC, et al. A common variant in the PNPLA3 gene is a risk factor for nonalcoholic fatty liver disease in obese Taiwanese children. J Pediatr. 2011;158:740-4.

37. Valenti L, Alisi A, Galmozzi E, et al. I148M patatin-like phospholipase domain-containing 3 gene variant and severity of pediatric nonalcoholic fatty liver disease. Hepatology. 2010;52:1274-80.

38. Liu YL, Patman GL, Leathart JB, et al. Carriage of the PNPLA3 rs738409 C > G polymorphism confers an increased risk of nonalcoholic fatty liver disease associated hepatocellular carcinoma. J Hepatol. 2013;61:75-81.

39. Seko Y, Sumida Y, Tanaka S, et al. Development of hepatocellular carcinoma in Japanese patients with biopsy-proven nonalcoholic fatty liver disease: association between PNPLA3 genotype and hepatocarcinogenesis/fibrosis progression. Hepatol Res. 2017;47(11):1083-92.

40. Singal AG, Manjunath H, Yopp AC, et al. The effect of PNPLA3 on fibrosis progression and development of hepatocellular carcinoma: a meta-analysis. Am J Gastroenterol. 2014;109:325-34.

41. Speliotes EK, Butler JL, Palmer CD, et al. PNPLA3 variants specifically confer increased risk for histologic nonalcoholic fatty liver disease but not metabolic disease. Hepatology. 2010;52:904-12.

42. Romeo S, Sentinelli F, Dash S, et al. Morbid obesity exposes the association between PNPLA3 I148M (rs738409) and indices of hepatic injury in individuals of European descent. Int J Obes (Lond). 2010;34:190-4.

43. Dubuquoy C, Robichon C, Lasnier F, et al. Distinct regulation of adiponutrin/PNPLA3 gene expression by the transcription factors ChREBP and SREBP1c in mouse and human hepatocytes. J Hepatol. 2011;55:145-53.

44. Huang Y, Cohen JC, Hobbs HH. Expression and characterization of a PNPLA3 protein isoform (I148M) associated with nonalcoholic fatty liver disease. J Biol Chem. 2011;286:37085-93.

45. Pingitore P, Pirazzi C, Mancina RM, et al. Recombinant PNPLA3 protein shows triglyceride hydrolase activity and its I148M mutation results in loss of function. Biochim Biophys Acta. 2014;1841:574-80.

46. Pirazzi C, Valenti L, Motta BM, et al. PNPLA3 has retinyl-palmitate lipase activity in human hepatic stellate cells. Hum Mol Genet. 2014;23:4077-85.

47. Kadowaki T, Yamauchi T. Adiponectin and adiponectin receptors. Endocr Rev. 2005;26:439-51.

48. Valenti L, Rametta R, Ruscica M, et al. The I148M PNPLA3 polymorphism influences serum adiponectin in patients with fatty liver and healthy controls. BMC Gastroenterol. 2012;12:111.

49. Ramezani-Moghadam M, Wang J, Ho V, et al. Adiponectin reduces hepatic stellate cell migration by promoting tissue inhibitor of metalloproteinase-1 (TIMP-1) secretion. J Biol Chem. 2015;290:5533-42.

50. BasuRay S, Smagris E, Cohen J, Hobbs HH. The PNPLA3 variant associated with fatty liver disease (I148M) accumulates on lipid droplets by evading ubiquitylation. Hepatology. 2017;66:1111-24.

51. Sevastianova K, Kotronen A, Gastaldelli A, et al. Genetic variation in PNPLA3 (adiponutrin) confers sensitivity to weight loss-induced decrease in liver fat in humans. Am J Clin Nutr. 2011;94:104-11.

52. Shen J, Wong GL, Chan HL, et al. PNPLA3 gene polymorphism and response to lifestyle modification in patients with nonalcoholic fatty liver disease. J Gastroenterol. 2015;30:139-46.

53. Valenti L, Dongiovanni P. Mutant PNPLA3 I148M protein as pharmacological target for liver disease. Hepatology. 2017;66:1026-8.
54. Liu YL, Reeves HL, Burt AD, et al. TM6SF2 rs58542926 influences hepatic fibrosis progression in patients with non-alcoholic fatty liver disease. Nat Commun. 2014;5:4309.

55. Holmen OL, Zhang H, Fan Y, et al. Systematic evaluation of coding variation identifies a candidate causal variant in TM6SF2 influencing total cholesterol and myocardial infarction risk. Nat Genet. 2014;46:345-51.

56. Dongiovanni P, Petta S, Maglio C, et al. Transmembrane 6 superfamily member 2 gene variant disentangles nonalcoholic steatohepatitis from cardiovascular disease. Hepatology. 2015;61:506-14.

57. Beer NL, Tribble ND, Mcculloch LJ, et al. The P446L variant in GCKR associated with fasting plasma glucose and triglyceride levels exerts its effect through increased glucokinase activity in liver. Hum Mol Genet. 2009;18:4081-8.

58. Valenti L, Alisi A, Nobili V. Unraveling the genetics of fatty liver in obese children: additive effect of P446L GCKR and I148M PNPLA3 polymorphisms. Hepatology. 2012;55:661-3.

59. Anstee QM, Darlay R, Leathart J, et al. A candidate gene approach to validation of genetic modifier associations using a large cohort with histologically characterised non-alcoholic fatty liver disease. J Hepatol. 2013;58:S46.

60. Petta S, Miele L, Bugianesi E, et al. Glucokinase regulatory protein gene polymorphism affects liver fibrosis in non-alcoholic fatty liver disease. PLoS One. 2014;9:e87523.

61. Zain SM, Mohamed Z, Mohamed R. Common variant in the glucokinase regulatory gene rs780094 and risk of nonalcoholic fatty liver disease: a meta-analysis. J Gastroenterol Hepatol. 2015;30:21-7.

62. Dongiovanni P, Valenti L, Rametta R, et al. Genetic variants regulating Insulin Receptor signaling are associated with the severity of liver damage in patients with nonalcoholic fatty liver disease. Gut. 2010;59:267-73.

63. Pawlak M, Lefebvre P, Staels B. Molecular mechanism of PPAR $\alpha$ action and its impact on lipid metabolism, inflammation and fibrosis in non-alcoholic fatty liver disease. J Hepatol. 2015;62:720-33.

64. Chen S, Li Y, Li S, Yu C. A Val227Ala substitution in the peroxisome proliferator activated receptor alpha (PPAR alpha) gene associated with non-alcoholic fatty liver disease and decreased waist circumference and waist-to-hip ratio. J Gastroenterol Hepatol. 2008;23:1415-8.

65. Di Rosa M, Malaguarnera L. Genetic variants in candidate genes influencing NAFLD progression. J Mol Med (Berl). 2012;90:105-18.

66. Dongiovanni P, Rametta R, Fracanzani AL, et al. Lack of association between peroxisome proliferator activated receptors alpha and gamma2 polymorphisms and progressive liver damage in patients with non-alcoholic fatty liver disease: a case control study. BMC Gastroenterol. 2010;10:102.

67. Dongiovanni P, Valenti L. Peroxisome proliferator-activated receptor genetic polymorphisms and nonalcoholic fatty liver disease: any role in disease susceptibility? PPAR Res. 2013;2013:452061.

68. Kumari M, Schoiswohl G, Chitraju C, et al. Adiponutrin functions as a nutritionally regulated lysophosphatidic acid acyltransferase. Cell Metab. 2012;15:691-702.

69. Valenti L, Motta BM, Alisi A, et al. LPIN1 rs13412852 polymorphism in pediatric nonalcoholic fatty liver disease. J Pediatr Gastroenterol Nutr. 2012;54:588-93.

70. Wiedmann S, Fischer M, Koehler M, et al. Genetic variants within the LPIN1 gene, encoding lipin, are influencing phenotypes of the metabolic syndrome in humans. Diabetes. 2008;57:209-17. 